\title{
Observer le pouvoir.
}

Pour une ethnographie du pouvoir dans le culte d'au Bénin. Corps du chercheur/Corps de l'Autre

Julie Poirée

\section{(2) OpenEdition}

1 Journals

\section{Édition électronique}

URL : http://journals.openedition.org/leportique/630

DOI : $10.4000 /$ leportique.630

ISSN : $1777-5280$

Éditeur

Association "Les Amis du Portique"

Édition imprimée

Date de publication : 1 septembre 2004

ISSN : 1283-8594

Référence électronique

Julie Poirée, "Observer le pouvoir. », Le Portique [En ligne], 13-14 | 2004, mis en ligne le 15 juin 2007, consulté le 25 mars 2021. URL : http://journals.openedition.org/leportique/630 ; DOI : https://doi.org/ 10.4000/leportique.630

Ce document a été généré automatiquement le 25 mars 2021.

Tous droits réservés 


\section{Observer le pouvoir.}

Pour une ethnographie du pouvoir dans le culte d'au Bénin. Corps du chercheur/Corps de l'Autre

\section{Julie Poirée}

Contexte et objet de recherche

2 En quelques décennies, le Bénin est devenu une nation officiellement catholique, mais il connaît cependant un développement original de son univers religieux. En effet, initialement fondés sur une volonté d'affirmation identitaire liée à la période de libération et d'indépendance des colonies, les mouvements religieux issus de mélanges entre christianisme missionnaire et cultes coutumiers sont en perpétuelle croissance. Parallèlement à ces phénomènes d'acculturation, se développe un pluralisme de l'offre religieuse - bien souvent à vocation thérapeutique - qui fait se côtoyer dans une même rue de la capitale économique temples protestants, mosquées et couvents initiatiques vodun ${ }^{1}$. Autant de structures religieuses dont la fonction sociale et identitaire est associée depuis une vingtaine d'années par les anthropologues, peut-être de manière quelque peu réductrice, au paradoxe moderniste : une industrialisation censée apporter développement et emploi, et la persistance d'une misère sociale associée à la montée d'un individualisme qui désintègre la cohésion communautaire. Au cœur de ce paysage cultuel bigarré, le culte d'Ethron Kpeto Deka Alafia, importé du Ghana au milieu du siècle dernier, est aujourd'hui le résultat d'une rencontre entre plusieurs traditions religieuses (pratiques religieuses ashanti et ewe, vodun béninois, christianisme et islam). Introduit au Bénin par, semble-t-il, un seul et unique hounnogan ${ }^{2}$, Tchakpé Tchedji, le culte est en pleine expansion et ne cesse d'évoluer. Chaque détenteur de ce fétiche ${ }^{3}$ construit son culte comme il l'entend, l'acquiert lorsqu'il le souhaite et le peut dans son parcours de vie, ou l'hérite directement de son père, tout en respectant certaines bases symboliques et pratiques du culte originel, tel que le premier importateur officiel l'a proposé après avoir traversé le Togo voisin.

3 Alafia est connu sur tout le littoral du Golfe de Guinée pour son pouvoir anti-sorcellerie, sa puissance thérapeutique en matière de fécondité, et sa capacité d'action dans les domaines de la réussite sociale et de la prospérité financière. Le culte rendu à ce Goro Vodun ${ }^{4}$ est constitué par d'impressionnantes offrandes de noix de cola à ce qui 
matérialise la divinité : un monticule de ces mêmes noix (entières et en cotylédons) au milieu d'une enceinte circulaire en plâtre ou en béton blanchi à la chaux. La principale caractéristique d'une telle construction est qu'elle s'édifie et s'améliore en fonction du pouvoir du détenteur et de ses moyens financiers. En d'autres termes, plus le hounnogan monte en grade, grâce au succès de ses activités magico-religieuses et à sa notoriété, plus il est en mesure financière et en droit par rapport à la coutume d'agrandir la maçonnerie consacrée au fétiche, de la consolider, de l'embellir par des ajouts de carrelage, allant parfois jusqu'au plafond du temple.

Actuellement, des conflits apparaissent entre représentants du culte: certains font preuve d'une créativité toute syncrétique en fondant des Églises d'Alafia (des messes au nom d'Alafia et des baptêmes sont organisés; la communion s'effectue non pas avec l'hostie mais avec un morceau de noix de cola), pendant que d'autres revendiquent l'authenticité de leur version rituelle qui oscille entre un polythéisme issu du panthéon vodun et un monothéisme où Alafia est vénéré comme un dieu suprême, universel et créateur de toute chose. La diversité des variantes du culte, outre qu'elle peut tout simplement correspondre à un processus classique de genèse religieuse, révèle néanmoins un contexte social où le religieux tient une place importante puisqu'il est manifestement soumis à une demande de la population en termes pratique, idéologique, symbolique, c'est-à-dire identitaires, et de facto à un éventail de réponses possibles, d'offres identitaires prenant pour les unes la forme d'une sorte de village reconstitué, celui qu'ils ont laissé derrière eux en venant chercher du travail à la ville, pour les autres un recours parmi d'autres aux problèmes de la vie quotidienne. Il y a bel et bien, aujourd'hui au Bénin, un marché du religieux, une concurrence dynamique entre les détenteurs du pouvoir sacré.

5 Ainsi apparaît-il nécessaire de comprendre cette nouvelle religiosité afin de saisir les évolutions de la société béninoise, mais également d'explorer le fonctionnement et la nature du pouvoir religieux. Si le pouvoir - thérapeutique, magique, social, politique $\mathrm{du}$ chef de culte attire une certaine population vers les temples, il faut aussi que ce pouvoir soit entretenu. Au Bénin, un temple peut disparaitre aussi rapidement qu'il est apparu par une désaffection progressive des adeptes envers le hounnogan et envers le lieu de culte, les deux étant étroitement liés, ainsi que j'ai commencé à l'expliquer plus haut et que je continuerai à le montrer plus bas. Mes enquêtes de terrain ont donc principalement porté sur la figure centrale du chef de culte, comme étant une des sources de construction et de maintien du culte, liée à une gestion des pouvoirs thérapeutique, social, politique, sacré, vus dans ce contexte comme des capacités qui s'acquièrent, s'entretiennent ou se perdent selon des modalités particulières.

Scènes de vie ou scénographie du pouvoir?

6 Jeudi 8 janvier 2004, 19 heures, maison Zannou, quartier Gankpodo, Cotonou, Bénin. J'attends le hounnogan Degbo Metonu depuis deux heures dans son salon. Nous avions rendez-vous à 17 heures. Deux rencontres au cours de la semaine dernière nous avaient permis de discuter de nos activités professionnelles respectives, et de la manière dont il pourrait m'aider à mieux comprendre le travail et la vie d'un chef de culte. Nous étions parvenus à la conclusion qu'il serait plus simple pour moi de « le regarder faire avec la divinité ». Sans qu'aucune allusion n'ait été faite de ma part à un quelconque appareil d'enregistrement, il m'avait dit, presque sous forme d'une demande, que je pourrais le photographier. Épuisée par la chaleur et le retard de Degbo, je tente de ne pas céder à l'énervement en sirotant tranquillement la quatrième bière fraîche que sa femme m'a 
gentiment apportée, et en suivant les oscillations du ventilateur qu'un des enfants de la maisonnée a rapproché, sans mot dire, de mon fauteuil. Après plusieurs enquêtes de terrain au Bénin et quelques courts séjours dans des couvents initiatiques, je ne suis toujours pas habituée à ce temps élastique, cette fameuse «heure africaine » que les Béninois manient, comme ils le disent eux-mêmes, avec humour et décontraction.

7 Trois jours plus tard (11 janvier 2004), le hounnogan Ogla Atcheffon, chef d'un temple d'Alafia dans la commune de Calavi, me fait attendre de la même manière, en respectant mon confort avec autant d'attention. L'heure du déjeuner arrive et une jeune fille m'apporte du riz, du poisson braisé et de la sauce à la tomate. Je mange seule dans le salon d'accueil quand j'aperçois mon hôte faire de même dans la pièce d'à côté. Je n'ose croire à de la désinvolture ou à un manque de considération de sa part. Cette attitude vis-à-vis du temps et de l'autre ne peut pas être uniquement une question de rythme, de décontraction ou d'humour.

8 Ce jeudi 8 janvier, persuadée que je vais assister à une prière devant l'autel dédié à Alafia, et connaissant déjà la configuration des lieux destinés aux actes rituels, ainsi que leur enchaînement dans le temps et l'espace, je prépare mentalement les angles de vue à privilégier en fonction des sources de lumière, les positions et déplacements autorisés dans le sanctuaire, les endroits où il me sera possible de poser mon magnétophone. Mais je me rends rapidement compte qu'aucun rituel, aucune libation aux fétiches, aucun priant en pagne blanc, aucune offrande d'adepte renouvelant sa confiance à Alafia, ne semblent prévus. J'assiste plutôt à une sorte de cérémonial d'habillage du hounnogan qui fait participer épouse, frères, sœurs et assistants.

Aidé de sa femme et de ses deux frères, Degbo choisit pendant un très long moment les plus beaux pagnes, enfile tous ses colliers, met un chapeau décoré des symboles d'Alafia, pour finalement reproduire parfaitement la tenue de cérémonie de son défunt père, dont le portrait peint à l'entrée du temple accueille tous les visiteurs. Degbo se place ensuite devant l'autel d'Alafia et me fait signe de le prendre en photo en train de saluer la divinité ; puis, il commande à son frère cadet un siège en bois, s'installe dessus et, toujours aidé de son frère, arrange le tombé de ses pagnes, sépare les colliers en les étalant sur sa poitrine, fixe le regard vers sa droite et me dit : « Tu peux y aller! Prends bien les pieds, prends-moi en entier ». S'ensuit une longue série de poses devant les endroits «forts» $d u$ temple: les fresques murales représentant la divinité anthropomorphisée, les autels d'Alafia et de son puissant mercenaire Bawungle ${ }^{5}$, et enfin la table où d'autres fétiches secondaires sont entreposés. Lorsque, entre deux prises de vues, je tente de photographier les couteaux rituels plantés sur le tas de noix de cola de l'autel principal, toute la famille, qui assiste à la scène, se met à rire; Degbo empoigne un couteau, le serre le long de son corps, bloque son coude replié contre le flanc gauche, je comprends alors qu'il pose à nouveau. Plus tard dans la journée, je souhaite faire différents clichés de la maçonnerie d'Alafia dénuée de toute mise en scène sociale, je demande alors à Degbo s'il est possible de photographier le fétiche, il accepte, mais pénètre soudainement le champ et s'appuie sur le rebord de l'autel afin de figurer sur les futures images avec son fétiche. Le malentendu est décidément total, ma liberté d'action formellement limitée.

10 La journée que je passe ensuite chez Ogla est en tout point similaire : l'habillage est long, soigné, objet de discussions et d'interventions familiales, les poses devant les fétiches s'enchaînent sans jamais laisser place à mes propres intentions photographiques. Que s'est-il passé lors de nos dernières entrevues pour que celle-ci 
tourne à une mise en scène qui n'a rien de commun avec les rituels habituellement consacrés à Alafia ? Devais-je revoir mon rapport à l'authenticité du culte et considérer qu'il se construit aussi dans des événements non rituels, quotidiens, voire improvisés ? Sans aucun doute.

11 À la fin de chaque "séance photos " - comment les appeler autrement? - les deux hounnongan me demandent de bien vouloir faire agrandir les photos, de les encadrer avec une baguette de qualité, et d'inscrire en bas des portraits leur nom rituel. Degbo a souhaité que je grave les photos sur un CD-Rom afin que les images ne se détériorent jamais.

Dans un premier temps, ces deux événements ont fait naître en moi le sentiment troublant d'avoir été utilisée, manipulée, instrumentalisée, transformée subtilement en support d'appareil photographique par un jeu de déplacements, de mouvements, de comportements, par le jeu des corps en interaction. L'objectif de l'ethnologue étant de dépasser la vexation procurée par ce genre d'expériences et de rendre le malentendu productif, une analyse moins " émotionnelle » permet de comprendre ces scènes de vie comme de véritables scénographies des pouvoirs impliquant féticheur et "observateur ": un retour réflexif sur les séances de prises de vue montre non seulement de quelle manière j'ai été prise et inscrite, comme tout autre ethnologue, dans un réseau particulier d'actions et d'influences, mais également de découvrir le fonctionnement, les moyens, les conditions pratiques et matérielles d'existence du pouvoir religieux.

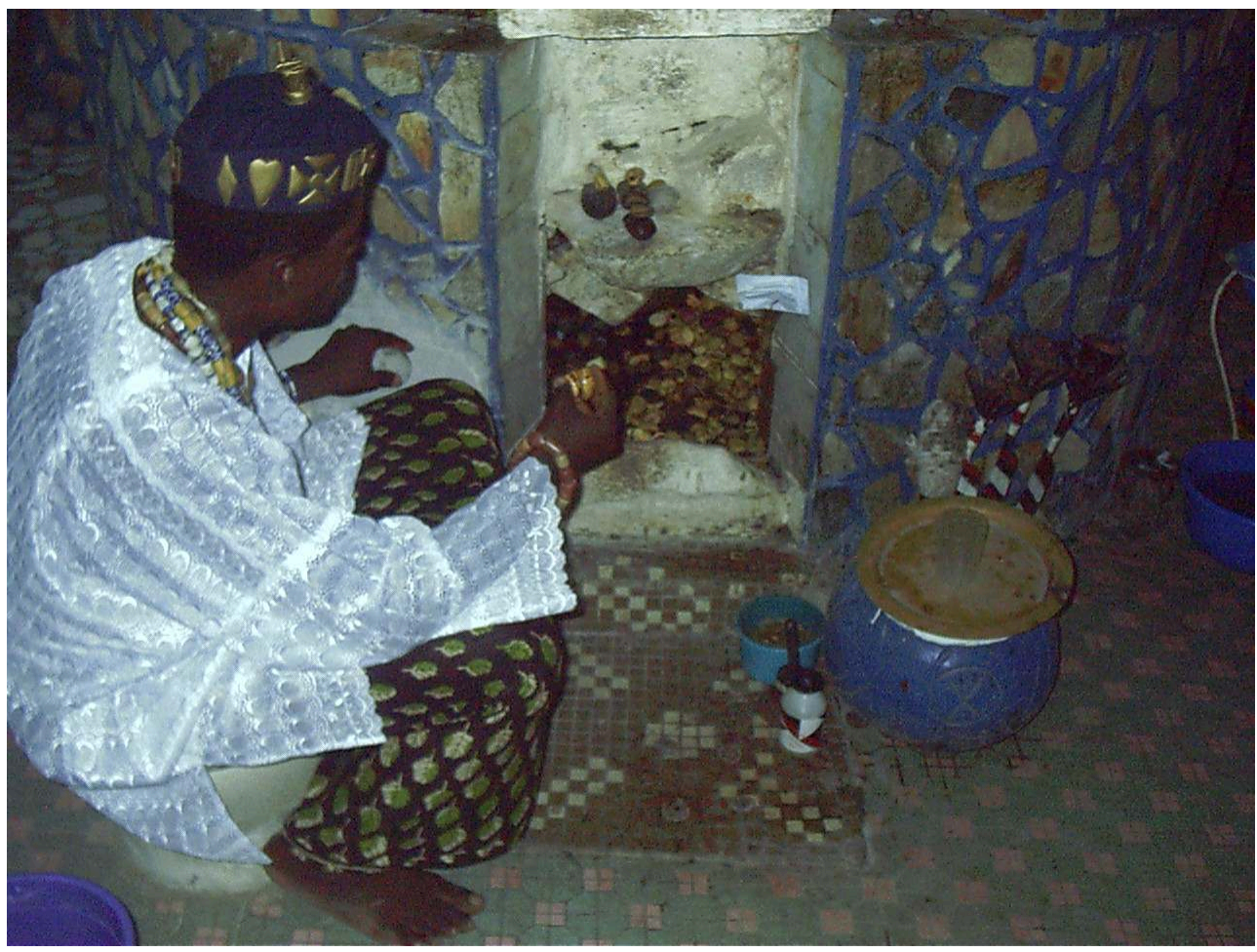

Hunnogan Degbo Metonu reproduisant une consultation d'Alafia

(photographie Julie Poirée) 


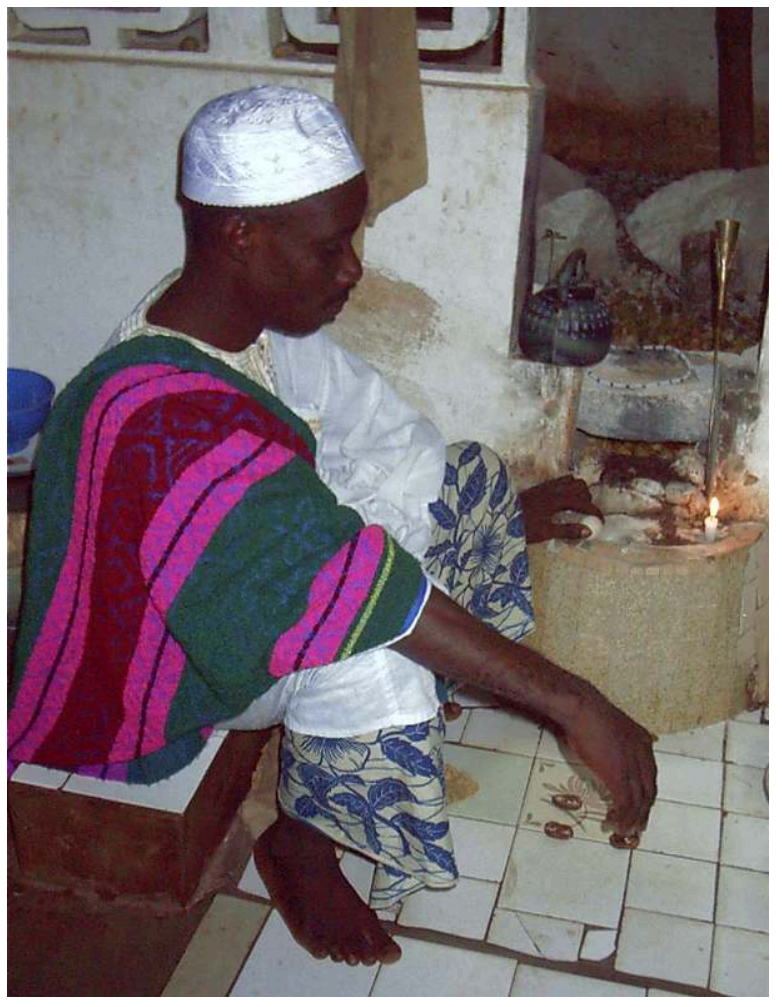

14 Hunnogan Ogla Atcheffon reproduisant une consultation d'Alafia (photographie Julie Poirée)

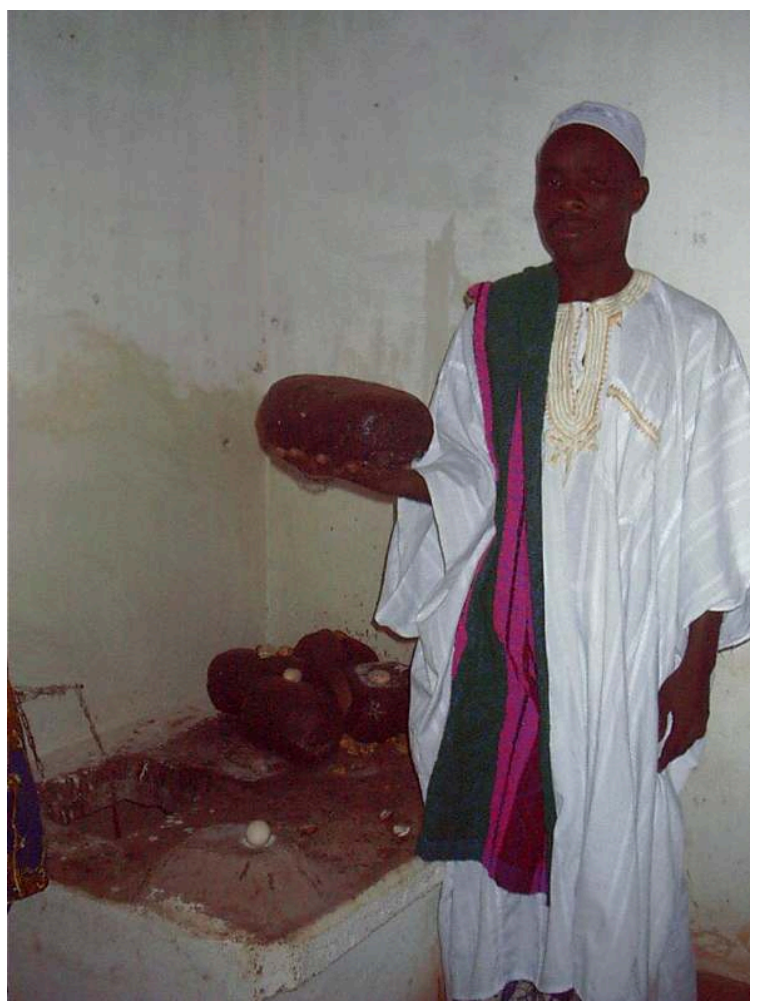

15 Hunnogan Ogla Atcheffon présentant une partie des vedun secondaires (photographie Julie Poirée)

La fabrication des pouvoirs. Le corps, l'espace, l'objet comme instruments de pouvoirLe corps et la culture matérielle du chef de culte 

de culte (porter des vêtements cérémoniels, avoir les pieds nus, ne jamais regarder l'objectif de l'appareil photo, être le plus souvent assis, être le moins mobile possible et déléguer les tâches aux membres de la famille) engendre du sens et du pouvoir. En effet, par l'intermédiaire des techniques du corps et d'objets, le chef de culte extériorise, exprime son pouvoir, et par là même le fabrique. Il y a comme une sorte d'ajout de pouvoir à chaque réaffirmation de celui-ci. Georges Balandier, dans le Détour, évoque ainsi les rapports qui existent entre le corps et le pouvoir du roi traditionnel africain : « il obéit à des obligations et interdits très stricts : de poids, de lenteur dans le mouvement, de posture, de secret dans l'accomplissement des fonctions vitales, d'impassibilité ou de "masque" (comme dans l'univers des civilisations du Bénin où des franges de perles dissimulent le visage royal), [...] d'émission de la parole, car les mots du roi ne circulent pas à l'identique des autres ${ }^{6}$. "Le souverain n'est libre de sa circulation ni à l'intérieur de l'enceinte palatiale (les espaces sont symboliquement qualifiés) ni à l'occasion de ses apparitions publiques. Certains contacts directs lui sont interdits parce qu'ils le déforcent, ou lui communiquent la souillure, ou l'attaquent en invisible " ${ }^{7}$. Le corps du roi produit du pouvoir par le marquage symbolique, par les impositions de comportement et par le rite. Dans certaines occasions, le pouvoir est donné à voir par une sorte de dramatisation cérémonielle. " Le corps royal, tout investi de signification, devient un support signifiant " et devient une "sorte de registre portant les inscriptions du pouvoir ${ }^{8}$. Les vêtements désignent le roi et changent selon les circonstances de sa manifestation publique. «Partout le roi est lié aux lieux sacrés du palais, aux regalia, à tout ce qui constitue, par l'extension de son corps physique, son " corps mystique ${ }^{9}$.

18 Aujourd'hui, au Bénin, les chefs de culte religieux ont comme remplacé les anciens rois (parfois même les anciens chefs de village) et remplissent des fonctions similaires mais à moindre échelle, c'est-à-dire qu'ils règnent non plus sur d'entières régions géographiques, mais sur des couvents initiatiques et des concessions familiales, à commencer par la leur. Ils ont en outre hérité d'une grande partie des codes comportementaux afférant au statut régalien. Ce qui peut expliquer certaines attitudes de Degbo et Ogla vis-à-vis de leur fétiche - dont la confection, rappelons-le, suit le parcours social et politique de son détenteur - et qui constituent pour eux la condition et l'extension physiques de leur personne. Par le corps et les actions rituelles du hounnogan, le fétiche peut agir sur la réalité ; de même, toutes les forces qui mettent en jeu le pouvoir du fétiche sont ressenties par le chef de culte. Le corps du fétiche est le corps du féticheur, le corps du féticheur est le fétiche. Tous deux se construisent et vivent conjointement, le pouvoir d'action thérapeutique et anti-sorcière du fétiche ne peut s'exercer et se maintenir sans le pouvoir politique, social et moteur du féticheur. "Les dieux et les rois occupent les positions clés du dispositif, alliés et imposant l'alliance de l'action rituelle et de l'action politique. La force du pouvoir est une résultante, le plus puissant des instruments de liaison dont disposent les hommes en vue de constituer une société en correspondance avec un monde naturel et surnaturel. Cette force est reconnue, nommée, commentée et mise en œuvre - mais par un seul, technicien de toutes les techniques, maitre de toutes les maitrises, le roi ou le chef ${ }^{10}$. 
Ainsi, «sa nature physique se manifeste comme une nature autre; elle porte signe, elle sera consacrée, elle peut révéler une élection par les dieux ou une identification à ceuxci " ${ }^{11}$. Balandier parle bien de "roi à faire» par des rituels de fabrication et d'incorporation du pouvoir. Il doit être «purifié, pleinement formé, marqué, identifié aux ancêtres et à la terre, et exprime la force du pouvoir et l'ordre qui en résulte " ${ }^{12}$. Une fois fabriqué, le roi est "présent avec un corps nouveau et à part qui signifie le pouvoir, physiquement par lui-même et symboliquement par ce qui le marque ou le revêt. " ${ }^{13}$.

19 L'idée d'une identification du féticheur à la divinité, d'une consubstantialité entre les deux «corps", conditionne tous les comportements et déplacements du hounnogan dans le sanctuaire dédié à Alafia, et de facto tout événement impliquant une mise en jeu $\mathrm{du}$ fétiche, comme celle provoquée par la séance de prises de vues. Le contact systématique avec les substances qui matérialisent l'existence de la divinité semble dans ce cas inévitable, évident.

20 Ainsi le féticheur se sent-il tenu, devant l'observateur extérieur, par rapport aux membres de sa famille et aux éventuels consultants susceptibles d'entrer dans le temple à tout moment, de réactualiser son pouvoir, non pas sa capacité d'action magico-thérapeutique, mais sa place dans la hiérarchie sociale, politique et religieuse. Bien que la raison principale d'une telle mise en scène soit l'opportunité d'être photographié, il n'en demeure pas moins que le féticheur doit respecter les contraintes comportementales, motrices, spatiales, matérielles liées à son statut sacré.

Le corps et la culture matérielle de l'ethnologue

L'observation des techniques d'objets et du corps du chef de culte pendant la séance de prises de vue ne peut à elle seule montrer les modalités de fonctionnement des pouvoirs en jeu dans cette situation. En effet, le retour sur mes propres conduites motrices durant ces deux journées semble nécessaire, car mon interminable attente dans les salons d'accueil n'est pas uniquement une question de rapport au temps mais de rapports de pouvoir, tel que Michel Foucault a voulu définir ce dernier. Sans contrainte directe, ni injonction violente, ni aucune autre forme de domination, de sujétion ou de coercition, Degbo et Ogla ont exercé et manifesté leur pouvoir, leur position dans la hiérarchie sociale, en me faisant attendre, en faisant participer femme et enfants à ce " réseau d'actions sur les actions des autres». Le corps est ainsi vecteur de pouvoir, il en reçoit et en produit. Le pouvoir se situe et naît dans la pratique et l'action, à travers l'utilisation des objets, la gestion des corps dans l'espace et le temps. La lecture des textes de Michel Foucault, notamment des Dits et écrits, permet à cet égard de "voir » le pouvoir dans ses formes complexes et plurielles. Selon l'auteur de l'article intitulé «Les mailles du pouvoir » ${ }^{14}$, il ne s'agit pas d'étudier «le » pouvoir car ce dernier n'est pas exercé par un seul. Ce qui caractérise le pouvoir est qu'il met en jeu des relations entre individus. Il est diffus, non localisable et traverse toute la société. Ces relations de pouvoir, dans lesquelles tout sujet est inscrit, ne se localisent pas dans les relations directes à l'État mais dans les corps. Elles sont partout dans une myriade de lieux par l'intermédiaire de différentes techniques. Avec Foucault, le pouvoir ne se donne plus comme une globalité transcendantale et pyramidale et n'implique pas forcément une domination d'un seul sur les autres, son exercice n'est pas simplement une relation entre des "partenaires ", individus ou collectifs, c'est un mode d'action de certains sur certains autres. 
Aussi, dans cette perspective, ne peut-il y avoir légitimation et réaffirmation du pouvoir qu'à travers la relation à l'autre, qu'à travers le rapport à un récepteur luimême acteur du pouvoir, en l'occurrence l'ethnologue. À travers cette «danse des corps » - c'est ainsi que j'ai appelé ces deux événements dans mon carnet de terrain -, le pouvoir du chef a trouvé les moyens pratiques de s'exprimer et de se réactualiser. Le positionnement physique du chef dans le temps et l'espace par rapport à celui de l'ethnologue correspond également à un positionnement symbolique et idéologique par rapport au statut présumé de ce dernier, une jeune femme occidentale implantée dans le milieu scientifique français et qui de surcroît semble maîtriser un savoir-faire technique reconnu, difficile d'accès et très prisé au Bénin, la photographie. Le culte d'Alafia étant en pleine période d'expansion et paradoxalement en position de fragilité par rapport aux autres religiosités instituées, il lui est nécessaire de se constituer une légitimée aux yeux de la population et des diverses autorités en place. Il est apparu au cours de mes enquêtes au Bénin que la pratique du portrait accroché au mur des pièces d'accueil était assez répandue chez les personnes à statut élevé, tels les médecins, les notables, les représentants administratifs, les dignitaires religieux. De même que tout document attestant la formation ou la position hiérarchique de l'intéressé, comme par exemple un diplôme de guérisseur traditionnel chez ogla Atcheffon ou une plaque en carton confectionnée en souvenir de l'intronisation de Degbo Metonu, survenue après la mort de son père. L'image photographique a donc ici un pouvoir de légitimation avéré, et l'outil de prise de vues est investi d'un sens différent selon son " utilisateur »: le hounnongan semble le considérer (et l'« utiliser ») comme un moyen de mise en scène de soi, alors que le chercheur s'en sert comme un instrument ethnographique producteur de connaissances scientifiques et de compréhension de l'Autre.

Si le savoir-faire et la culture matérielle de l'ethnologue (carnet de notes, appareil photographique, vêtements, moyens financiers) sont incontestablement les instruments visibles de son pouvoir sur le terrain, ils sont également des éléments sur lesquels le chef de culte peut/doit avoir une prise pour manifester et asseoir son propre pouvoir, ne serait-ce que vis-à-vis des observateurs que sont les membres de sa famille, les adeptes et consultants présents dans le temple, ainsi que les futurs visiteurs (patients, collègues, dignitaires religieux, voire représentants politiques) qui verront accrochés sur le mur du salon une série de portraits en pieds du hounnongan.

Ainsi, il semble intéressant d'élargir la panoplie des instruments de pouvoir du chef de culte à tout ce qui ne fait pas a priori partie de son propre univers matériel, pratique et symbolique. À chaque instant, le hounnongan « se situe " par rapport à cette région de pouvoir en mouvement que représente l'ethnologue et entre dans un jeu avec ses objets, ses techniques du corps et ses intentions. Le corps de l'ethnologue, sa culture matérielle, son savoir-faire, et son statut sont en quelque sorte utilisés et intégrés dans la panoplie des techniques de pouvoir du chef. L'ethnologue est bel et bien pris dans le dispositif de pouvoir religieux que le chef de culte est censé maintenir et entretenir. Mais «pris » ne signifie pas pour autant totalement agi et manipulé; je dirais plutôt que l'ethnologue est engagé en tant qu'acteur d'une scénographie des pouvoirs, même si, sur le moment, je ne me souviens pas avoir été réellement maîtresse de la situation et consciente de l'emprise que chaque féticheur avait sur mon jeu d'actrice. 

d'une dramatisation cérémonielle, il fait allusion à des techniques de mise en scène du pouvoir qui se déploient dans des occasions particulières, telles les intronisations, les rituels de renforcement de l'autorité du souverain, les fêtes annuelles liées dans certaines traditions à la récolte de l'igname. Le pouvoir du chef est sans cesse à fabriquer et à pérenniser par un ensemble de pratiques rituelles spécifiques, de techniques programmées, de contraintes corporelles et symboliques censées assurer son maintien. Ce chef fabriqué « est le résultat de tout un travail et le travail du souverain est de se conformer à une programmation qui le constitue en opérateur social central; il a la charge des relations avec le monde et les principales puissances symboliques, il doit tenir la société dans l'unité et la préserver de tout déforcement ». sacré (qui concernent le chef de culte tout autant que le roi), il me semble que l'on peut également voir dans les mises en scène des deux hounnongan des techniques destinées à remplir la même fonction de maintien. Celles-ci s'inscrivent dans un contexte plus quotidien, plus privé, et certes en marge des manifestations régulières du pouvoir sacré, mais n'en sont pas moins des pratiques de renouvellement, de reproduction et d'incorporation de ce dernier. Albert Piette parlerait peut-être d'un "mode mineur " de fabrication des pouvoirs pour décrire ces techniques profanes d'actualisation du pouvoir, mais ce « mode mineur de la réalité ${ }^{15}$, c'est-à-dire quelque peu écarté de la règle établie, ne participe pas moins à sa construction que les événements prévus et organisés.

L'objectif de cette présente analyse était de mettre certains outils conceptuels foucaldiens au service non seulement de la réflexion mais de l'observation ethnologique. Tenter d'identifier le pouvoir au sein de la société ou d'un groupe d'individus n'est en effet pas chose aisée. En lectrice bricoleuse-fouilleuse des Dits et écrits, je me suis appropriée un des parcours possibles que propose Foucault, celui des techniques corporelles de pouvoir. J'ai tenté de voir, de repérer le pouvoir mis en action, mis en objet et mis en corps, afin de saisir le « comment » du pouvoir.

Toute rencontre individuelle est négociation identitaire et production de pouvoir. L'ethnologue est sans cesse en train de négocier sa présence, sa place sur le terrain, de se situer physiquement par rapport à ses informateurs, et ne peut échapper à une implication corporelle dans les dispositifs de pouvoir et les processus de subjectivation que ses interlocuteurs lui imposent de fait. Le retour sur soi, l'analyse de son parcours intellectuel et physique de chercheur face à l'altérité sont dès lors des modes incontournables de saisie de la réalité observée.

\section{NOTES}

1.. Religion polythéiste pratiquée principalement dans les zones sud du Bénin et du Togo, et longtemps assimilée à du fétichisme en raison des cultes rendus aux divinités par l'intermédiaire d'objets divers, tels des figurines anthropomorphes ou des

Le Portique, 13-14 | 2004 
monticules de terre. Certains sanctuaires abritant les fétiches - ainsi que les Béninois les appellent eux-mêmes - sont consacrés à des séjours d'initiation plus ou moins longs et ont pris le nom à connotation chrétienne de « couvents ».

2.. Grand chef de culte traditionnel, en langue fon (parlée dans tout le sud du Bénin).

3.. Ce terme est utilisé par les chefs de culte eux-mêmes pour désigner la matérialisation de la divinité, $\mathrm{j}$ 'emploie donc ce vocable volontairement - même si les adeptes rendent leur culte non pas à l'objet mais à la divinité qu'il représente.

4.. Divinité de la cola (du terme ewe " goro » : noix de cola).

5.. Le culte d'Ethron Kpeto Deka Alafia se compose généralement d'un fétiche principal (Alafia), d'un fétiche mercenaire placé dans un sanctuaire à part (Bawungle) et dont la fonction est d'agir « en invisible " pour le compte d'Alafia, et parfois d'un troisième fétiche considéré comme l'épouse d'Alafia (Nana Blewa).

6.. Georges BALANDIER, Le Détour. Pouvoir et modernité, Paris, Fayard, «L’Espace du Politique », 1985, p. 35.

7.. Ibid.

8.. Ibid., p. 36.

9.. Ibid.

10.. Ibid.

11.. Ibid., p. 34.

12.. Ibid.

13.. Ibid., p. 35.

14.. Michel FOUCAULT, «Les mailles du pouvoir », Dits et écrits, T. II, Gallimard, Quarto, 2003, p. 1001.

15.. A. PIETTE, Ethnographie de l'action. L'observation des détails, Paris, Métailié, 1996.

\section{RÉSUMÉS}

Le culte d'Ethron Kpeto Deka Alafia, issu de la rencontre entre christianisme missionnaire, islam et pratiques coutumières, se répand avec succès au Bénin par l'intermédiaire de meneurs dont le pouvoir magico-religieux, thérapeutique, social et politique doit être acquis et sans cesse entretenu pour la pérennité d'une tradition en pleine genèse. Les outils conceptuels proposés par Michel Foucault pour identifier les formes que prend l'exercice du pouvoir, pour le "voir» émerger au sein des rapports sociaux, sont susceptibles d'orienter l'observation et l'analyse ethnologiques. Le pouvoir naît et se déploie dans l'action rituelle mais également dans la pratique quotidienne. Le pouvoir du chef de culte, par exemple, s'exprime par diverses voies, rarement mises en corrélation, celles du corps, de l'espace, de la matière et du rapport à autrui.

The worship of Ethron Kpeto Deka Alafia, which is a combination of elements from Christianity, Islam and traditional religions, is successfully spreading all over Benin, through the work of leaders whose powers - magic, religious, therapeutic, social and political - have to be acquired and endlessly fed in order to allow this cult in progress to endure. Michel Foucault's conceptual framework to identify the various forms of power and "see" it appear in social relations can give bearings for anthropological observation and analysis. Power comes from, and grows through, ritual acts and daily individual practices. For instance, the power of the religious leader 
manifests itself by different means which are rarely related: the body, the space, the material culture and the relations with other people. 